\title{
Localized Chondrosarcoma
}

National Cancer Institute

\section{Source}

National Cancer Institute. Localized Chondrosarcoma. NCI Thesaurus. Code C8778.

A non-disseminated skeletal or extraskeletal chondrosarcoma. 